\title{
Die Grundkonzepte der Pathologie in Deutschland von 1858 bis heute und der Fortschrittsbegriff in der Medizin
}

Cay-Rüdiger Prüll

\section{Summary}

The rise of scientific medicine in the 19th century had its origins mainly in Rudolf Virchows localistic cellular pathology. As a consequence the organism as a complex system was kept in the background. In recognition of this problem, concepts of pathology, emerging in 20th century, tried in vain to establish organismic theories of illness. Pathology remained deeply indebted to Virchows work. Deficits appeared even in 19th century, when treatment of patients was mainly focussed on practicability of cure, ignoring the social background. Therefore, it is not possible to speak about progress of pathology in general, for diagnostics depends also on individual mentality, the subject, and the situation of the time.

\section{Zusammenfassung}

Die Entwicklung der lokalistischen Zellularpathologie Rudolf Virchows im 19. Jahrhundert war grundlegend für die Entstehung der naturwissenschaftlichen Medizin, die Sicht auf den Gesamtorganismus trat dabei in den Hintergrund. Dies konnte im 20. Jahrhundert durch ganzheitliche Konzeptionen nicht geändert werden, da die Pathologie der lokalistischen Sehweise Virchows verpflichtet blieb. Die Defizite zeigten sich schon im 19. Jahrhundert in der Patientenbehandlung, die unter Ausblendung der sozialen Situation des Kranken vorwiegend dem Machbarkeitskriterium unterlag. Daher kann nicht von einem allgemeinen Fortschritt in der Geschichte der Patho-

Dr. med. Cay-Rüdiger Prüll M.A., Institut für Geschichte der Medizin, Albert-Ludwigs-Universität Freiburg, Stefan-Meier-Strasse 26, D-79104 Freiburg i.Br. 
logie gesprochen werden. Eine Bewertung von Fortschrittlichkeit ist vielmehr abhängig von der Einstellung des betroffenen Subjektes, dem Gegenstand der vermeintlichen oder tatsächlichen Fortschrittlichkeit und der historischen Situation.

\section{Einleitung}

Für die Medizin ist die Frage nach «Fortschritten» in der Heilkunde und «fortschrittsorientiertem Handeln» heute eine systemimmanente Notwendigkeit. Praktizierende, forschende und lehrende Ärzte hatten freilich - seit es durch den Historiker aufgrund schriftlicher Quellen nachvollziehbar ist das Bedürfnis nach der Einordnung ihres Handelns in zeitliche Zusammenhänge. Damit sollte es ermöglicht werden, die Bedeutung der jeweils aktuellen Entwicklung des Faches vor dem Hintergrund der Vergangenheit einschätzen zu können.

Die Frage nach «Fortschrittlichkeit» wurde allerdings dringlicher, nachdem sich die naturwissenschaftliche Medizin seit der Mitte des 19. Jahrhunderts gegenüber bisher dominierenden Richtungen in der Heilkunde durchgesetzt hatte und sich im Angesicht ihrer Erfolge als Endpunkt einer Geschichte des Fortschritts in der Medizin präsentierte. So schrieb Emil Du Bois-Reymond: «Wir sagen, Naturwissenschaft ist das absolute Organ der Cultur, und die Geschichte der Naturwissenschaft die eigentliche Geschichte der Menschheit» ${ }^{1}$. Dabei entsprach diese Einschätzung der zeitgenössischen wissenschaftstheoretischen Beurteilung, die «die Weiterentwicklung der Gattung Mensch» in Abkehr von der praktisch-philosophischen Bestimmung des Fortschrittsbegriffes des 18. Jahrhunderts «in den unbegrenzten technischen Möglichkeiten der Naturbeherrschung» sah ${ }^{2}$. Die Verbreitung des Fortschrittsbegriffes in der Medizin um die Mitte des 19. Jahrhunderts soll nur anhand des Zeitschriftenwesens angedeutet werden. Ab 1842 hatte Carl Friedrich Canstatt (1807-1850) in Erlangen den «Jahresbericht über die Fortschritte der gesammten Medicin in allen Ländern» herausgegeben. Unter der Herausgeberschaft von Rudolf Virchow (1821-1902) und August Hirsch (1817-1894) zwischen 1867 und 1888 wurde die Zeitschrift dann, den Charakter der Progressivität noch stärker hervorhebend, in «Jahresberichte

1 Vgl. Emil Du Bois-Reymond, Culturgeschichte und Naturwissenschaft (Vortrag, gehalten am 24. März 1877 im Verein für wissenschaftliche Vorlesungen zu Köln), Leipzig 1878, S. 35.

2 Zitate aus Jürgen Mittelstrass, «Fortschritt», in: ders. (Hg.) Enzyklopädie, Philosophie und Wirtschaftstheorie, Bd. 1, Mannheim/Wien/Zürich 1980, S. 664-666, hier S. 665. Siehe auch Joachim Ritter, «Fortschritt», in: ders. (Hg.), Historisches Wörterbuch der Philosophie, Bd. 2, Basel/Darmstadt 1972, Sp. 1032-1059. 
über die Leistungen und Fortschritte in der gesammten Medicin» umbenannt ${ }^{3}$. Der zeitgenössische Optimismus der Medizin, der aus Leistungen Fortschrittsglauben entstehen liess, zeigt sich am Verhalten eines ihrer prominentesten Vertreter, das geradezu Symbolcharakter für das gesamte 19. Jahrhundert hat: Nur ein Jahr, nachdem der Hygieniker Robert Koch (1843-1910) den Erreger der Tuberkulose entdeckt hatte, war er im Jahre 1883 Mitbegründer der Zeitschrift «Fortschritte der Medizin» als «Internationale Zeitschrift für die gesamte Heilkunde» ${ }^{4}$.

Für jene Fortschritte der naturwissenschaftlichen Medizin in der zweiten Hälfte des 19. Jahrhunderts spielte die Lehre und schliesslich das Spezialfach der Pathologie eine beträchtliche Rolle 5 . Zu Beginn des 19. Jahrhunderts wurde der Begriff «Pathologie» ausschliesslich im Sinne von «allgemeiner Krankheitslehre» gebraucht. Er war gleichsam ein Arbeitsbegriff, der von dem jeweiligen medizinischen Fachvertreter - dessen Theorien von Krankheit und Kranksein entsprechend - mit Inhalt gefüllt wurde ${ }^{6}$, bis sich nach 1800 auf dem Hintergrund des morphologischen Gedankens die Auffassung von Pathologie als pathologischer Anatomie durchsetzte. Mithilfe der routinemässigen Sektion führte die Umsetzung des morphologischen Standpunktes zunächst zum Gewinn zahlreicher Einzelerkenntnisse von Krankheiten, die unter dem Oberbegriff der «speziellen Pathologie» zusammengefasst und in Hand- und Lehrbüchern der Heilkunde zugänglich gemacht wurden. Aus dem erarbeiteten Einzel- und Faktenwissen ergab sich im Überblick die Möglichkeit, im Rahmen einer «allgemeinen Pathologie» den

3 Vgl. Walter A.L. v. Brunn, Das deutsche medizinische Zeitschriftenwesen seit der Mitte des 19. Jahrhunderts (Riedel-Archiv, Sonderheft), Berlin-Britz 1925; enthält abgesehen von dem falsch wiedergegebenen Titel verwertbare Daten über die Herausgeber und das weitere Schicksal der Zeitschrift. Siehe auch: ders., Medizinische Zeitschriften im 19. Jahrhundert. Beiträge zur Geschichte der allgemein-medizinischen Fachpresse, Stuttgart 1963, S. 81.

4 Vgl. Carl Friedländer, Prospectus, in: Fortschritte der Medicin 1 (1883) 1-3.

5 Die wichtigsten Publikationen zur Geschichte der Pathologie: Russel C. Maulitz, The Pathological Tradition, in:W.F. Bynum, Roy Porter, Companion Encyclopedia of the History of Medicine, Vol. 1, London/New York 1993, S. 169-191; George J. Cunningham, The History of British Pathology, Bristol 1993; Axel Bauer, Die Krankheitslehre auf dem Weg zur naturwissenschaftlichen Morphologie. Pathologie auf den Versammlungen Deutscher Naturforscher und Ärzte von 1822-1872, Stuttgart 1989; Russel C. Maulitz, Morbid appearances. The anatomy of pathology in the early nineteenth century, Cambridge 1987; Teizo Ogawa (Hg.), History of Pathology. Proceedings of the 8th international Symposium on the comparative history of Medicine - East and West (18.-24. Sept. 1983, Susono-shi, Shizuoka, Japan), Tokyo 1983; William Derek Foster, Pathology as a profession in Great Britain and the early history of the Royal College of Pathologists, London 1983; ders., A short History of Clinical Pathology, Edinburgh/London 1961; E.B. Krumbhaar, Pathology (Clio Medica XIX), New York (1937) $1962<$ <achdr.>; Esmond R. Long, A History of American Pathology, Springfield (USA) 1962; ders., A History of Pathology (Baltimore 1928), New York 1965; W. Fischer, Georg B. Gruber, Fünfzig Jahre Pathologie in Deutschland, Stuttgart 1949; Edgar Goldschmid, Entwicklung und Bibliographie der pathologisch-anatomischen Abbildung, Leipzig 1925.

6 Bauer, Die Krankheitslehre, S. 21/22 (n. 5). 
Versuch zu unternehmen, generelle Eigenschaften von Krankheit nach dem jeweilig aktuellen Erkenntnisstand zusammenzustellen. Pathologie wurde demnach im Wesentlichen zur pathologischen Anatomie und zur allgemeinen Pathologie, wobei sich letztere aus ersterer ableiten lässt ${ }^{7}$.

In der vorliegenden Untersuchung soll die Entwicklung der Pathologie zwischen 1858 und heute im Hinblick auf den Fortschrittsgedanken untersucht werden. Vor dem Hintergrund der Entwicklung der Zellularpathologie von Rudolf Virchow Mitte des letzten Jahrhunderts schuf das Fach die Grundlage für die moderne kausalanalytisch-naturwissenschaftliche Heilkunde. Die wesentlichen Strömungen der Pathologie - Zellularpathologie, Konstitutionspathologie, Molekularpathologie sowie die Theoretische $\mathrm{Pa}-$ thologie - sollen im Folgenden vorgestellt, ihre Rolle im Hinblick auf «Fortschritte» der Medizin anschliessend analysiert werden. Abschliessend ist auf der Grundlage der gewonnenen Ergebnisse der Fortschrittsbegriff für die naturwissenschaftliche Medizin zu präzisieren.

\section{Rudolf Virchow und die Zellularpathologie als «fortschrittliches Prinzip»}

Im Jahre 1858 führte der Berliner Pathologe Rudolf Virchow ${ }^{8}$ (1821-1902) mit seinem Werk «Die Cellularpathologie in ihrer Begründung auf physiologische und pathologische Gewebelehre» ${ }^{9}$ das «cellulare Princip» in die Medizin ein. In deutlicher Abwendung von diversen, noch in seiner Zeit erfolgreichen medizinischen Systemen, vor allen Dingen der Humoralpathologie, die ihr Krankheitskonzept auf die Eu- bzw. Dyskrasie der ungeformten Kör-

7 Vgl. Walter Sandritter, Pathologie, in: Eduard Seidler (Hg.), Wörterbuch medizinischer Grundbegriffe. Eine Einführung in die Heilkunde in 86 Artikeln, Freiburg 1979, S. 236-238; ders., Das Selbstverständnis der Pathologie, in: Die Medizinische Welt 29 (1978) 1507-1510; E. Randerath, Pathologie als praktisches und wissenschaftliches Problem, in: Münchner Medizinische Wochenschrift 97 (1955) 65-70; K. Rudinski, Überlastete Pathologie, in: FAZ v. 9.6.1976 (Nr. 123), S. 31/32. Für Axel Bauer bilden ebenfalls morphologische Untersuchungsmethoden die Grundlage der Pathologie. Die angeführten Definitionen namhafter Pathologen werden allerdings keiner weitergehenden Analyse unterzogen. Auch wird der Tatbestand vernachlässigt, dass auch die pathologische Anatomie nie ohne ein theoretisches Konzept auskommen konnte. Vgl. ders., Die Krankheitslehre, S. 7-10 (n. 5).

8 Die wichtigsten Werke zu Virchow: Erwin H. Ackerknecht, Rudolf Virchow. Arzt, Politiker, Anthropologe, Stuttgart 1957; Manfred Vasold, Rudolf Virchow. Der grosse Arzt und Politiker, Stuttgart 1988; Christian Andree, Rudolf Virchow, in:Wilhelm Treue, Rolf Winau (Hrsg.), Mediziner (Berlinische Lebensbilder 2) Berlin 1987, S. 175-190; Heinz David, Rudolf Virchow und die Medizin des 20. Jahrhunderts, hrsg.v. Werner Selberg u. Hans Hamm (Hamburger Beiträge zur Geschichte der Medizin, hrsg.v. W.S. u. H.H.), München 1993.

9 Rudolf Virchow, Die Cellularpathologie in ihrer Begründung auf physiologische und pathologische Gewebelehre. Zwanzig Vorlesungen, gehalten während der Monate Februar, März und April 1858 im pathologischen Institut zu Berlin, Berlin 1858. 
perelemente stützte, postulierte Virchow in Fortsetzung solidarpathologischen Gedankengutes die Bedeutung der Zelle für Gesundheit und Krankheit in ihrer Eigenschaft als Grundbaustein des menschlichen Körpers. Dabei ist die Zelle nicht nur Träger der physiologischen Körperfunktionen, sondern ebenfalls Schauplatz der Erkrankungen des Körpers. Krank ist demnach für Virchow nicht mehr der ganze Körper, sondern vielmehr einzelne Zellen beziehungsweise Zellverbände ${ }^{10}$. Die Frage nach dem Wesen der Krankheiten wird von Virchow im Jahre 1856 wie folgt beantwortet: «Für uns ist diese «Frage, s.o.> sehr einfach. Das pathologische Wesen ist die kranke Zelle und die Krankheit hat keine andere Einheit, als das Leben, von dem sie nur eine besondere Art darstellt; nämlich die einheitlich lebende Zelle. Das Leben der kranken und der gesunden Zelle unterscheidet sich nicht dadurch, dass bloss die äusseren Bedingungen andere sind, oder dass die Zelle eine Störung erfahren hat..., sondern durch den Charakter der Gefahr, den das Leben der erkrankten Zelle an sich trägt» ${ }^{11}$.

Entgegen den Stimmen zahlreicher Kritiker und trotz eigener kritischer Betrachtungen bezüglich eines «Krankheitswesens» ${ }^{12}$ behielt Virchow diese Auffassung im Prinzip bei. So schrieb er 1880: «Allen diesen Richtungen gegenüber stellte ich die lebende Zelle als das eigentliche Krankheitswesen auf oder, wie ich es zuerst ausdrückte..., ich definirte die kranke Zelle als das pathologische Wesen (ens morbi).» ${ }^{13}$, dann später: «Wem die Auffassung der veränderten Zelle als des Ens morbi nicht passt, der sollte uns sagen, was er an die Stelle setzt» ${ }^{14}$. Dementsprechend noch 1895: «Immer wird die Krankheit als ein veränderter Lebenszustand einer kleineren oder grösseren Zahl

10 Vgl. Ackerknecht, Rudolf Virchow, S. 58ff. (n. 8); Otto Helfer, Rolf Winau (Hrsg.), Männer und Frauen der Medizin. Illustrierte Kurzbiographien zur Geschichte der Medizin, Berlin/New York 1986, S.86; Rudolf Virchow, Cellular-Pathologie, in: Archiv für pathologische Anatomie und Physiologie und für klinische Medicin 8 (1855) 14/15. Zur Auseinandersetzung mit Kritikern: ders., Die Kritiker der Cellularpathologie, in: Archiv für pathologische Anatomie und Physiologie und für klinische Medicin 18 (1860) 1-14.

11 Rudolf Virchow, Alter und neuer Vitalismus, in: Archiv für pathologische Anatomie und Physiologie und für klinische Medicin 9 (1856), H. 1/2, S. 3-55, hier S. 54.

12 Ders., Über die heutige Stellung der Pathologie < Tageblatt der 43. Versammlung der Deutschen Gesellschaft für Naturforscher und Ärzte in Innsbruck 1869, Nr. 8, 25. Sept. 1869, S. 185-195. Wiederabgedruckt in: Karl Sudhoff, Rudolf Virchow und die Deutschen Naturforscherversammlungen, Leipzig 1922>, in: Karl Eduard Rothschuh, Was ist Krankheit? Erscheinung, Erklärung, Sinngebung, Darmstadt 1975, S. 72-91, hier S. 84: «Es gibt kein Wesen der Krankheit, sondern jede Krankheit ist etwas Besonderes für sich, und jede dieser Krankheiten kann nicht bloss, sondern muss in der Regel durch eine andere Ursache hervorgebracht sein; ...». Virchow widerspricht sich allerdings, wenn er auf S. 89 behauptet: «Denn zum Schlusse muss ich Ihnen sagen, dass ich persönlich und die, wie ich hoffe, immer zunehmende Zahl der Ärzte die Vorstellung haben, dass es ein Krankheitswesen gebe. Diese Erkenntnis ist gewonnen worden auf dem Wege der pathologischen Anatomie...».

13 Ders., Krankheitswesen und Krankheitsursachen, in:Archiv für pathologische Anatomie und Physiologie und für klinische Medizin 79 (1880), 7. Folge, Bd. 9, H. 1, S. 1-19, hier S. 5.

14 Ebda., S. 7. 
von Zellen oder Zellterritorien betrachtet werden müssen, und ob die Ursache der Krankheit im Blute cirkulirt oder direkt an die Zellen gelangt, wird das Urtheil über das Verhältniss der Ursachen zu den Zellen nicht ändern ${ }^{15}$.

Das neue «cellulare Prinzip» ${ }^{16}$, wie Virchow es nannte, wirkte sich auf die praktische und theoretische zeitgenössische Heilkunde insofern aus, als auf der Grundlage der von Virchow gleichfalls propagierten naturwissenschaftlichen Methodik ${ }^{17}$ die Weiterführung des morphologischen Gedankens zu einem extremen Lokalismus vollzogen wurde. Virchows Beschäftigung mit der Zelle lenkte die Aufmerksamkeit in Praxis und Forschung eher auf verschiedene Regionen des menschlichen Körpers als auf dessen Gesamtheit ${ }^{18}$.

Auf der Grundlage des zeittypischen Fortschrittsbegriffes («Die Zeiträume, welche der Mensch überblickt, ... gestatten ... uns, die befriedigende Überzeugung zu gewinnen, dass ein freilich sehr langsamer und häufig unterbrochener Fortschritt in der Welt ist.» ${ }^{19}$ ) betrachtete Virchow sein Prinzip dabei durchaus als Fortschritt. So bezeichnete er 1852 die neue Einteilung der Zellveränderungen bei krankhaften Zuständen deutlich als «Fortschritt gegen die früheren, ziemlich vagen Aufstellungen, die man unter Abweichungen der Farbe, der Cohäsion, der Textur etc. zusammenfasste und die jedes durchgreifenden Eintheilungsprincips, jeder Einheit der Anschauung entbehrten $»^{20}$. Zehn Jahre später, im Jahr 1862, wurde Virchow im Zusammenhang mit seiner Zellenlehre noch deutlicher, wenn er betonte: «Was man blos in der Idee gesucht hatte, das hat man endlich in der Wirklichkeit gefunden; was Vielen ein Traum erschien, das hat einen sichtbaren Leib gewonnen, es steht wahrhaftig vor unserem Auge da». Seiner Lehre mass er auch historische Bedeutung bei, wenn er betonte, die «Reformation» müsse «permanent sein», Inhalt und Fassung müsse «auch dem fortschreitenden Wissen nachgeben $»^{21}$. Virchow stellte sich bewusst in die Traditionskette von Giovanni Battista Morgagni (1682-1771), der den Sitz der Erkrankungen in

15 Ders., Hundert Jahre Allgemeiner Pathologie, Berlin 1895, S. 42.

16 Ders., Krankheitswesen und Krankheitsursachen, S. 16.

17 Ders., Unser Jubelband, in: Archiv für pathologische Anatomie und Physiologie und für klinische Medicin 150 (1897) 1-15; ders., Die naturwissenschaftliche Methode und die Standpunkte in der Therapie, in: Archiv für pathologische Anatomie und Physiologie und für klinische Medicin 2 (1849) 3-37.

18 Siehe zu Virchows Ansichten über die Zelle auch: Rudolf Virchow. Sämtliche Werke, hrsg.v. Christian Andree, Bd. 4,Abt.I, Medizin. Beiträge zur wissenschaftlichen Medizin aus den Jahren 1846-1850, bearb.v. Christian Andree, Bern/Berlin u.a. 1992.

19 Virchow, Alter und neuer Vitalismus, S. 25.

20 Ders., Ernährungseinheiten und Krankheitsheerde, in: Archiv für pathologische Anatomie und Physiologie und für klinische Medizin 4 (1852) 375-399, hier S. 395.

21 Ders., Über die mechanische Auffassung des Lebens, in: ders., Vier Reden über Leben und Kranksein, Berlin 1862, S. 3-33, Zitate auf S. 8, 21. 
die Organe verlegt hatte, und François Xavier Bichat (1771-1802), der den Blick in Richtung von Organteilen, nämlich den Geweben vertieft hatte ${ }^{22}$. Damit war die Pathologiegeschichte eingebettet in einen Fortschrittsglauben, der die gesamte Medizingeschichte betraf: «Man kann darin die Geschichte des Fortschritts aufzeichnen; man kann sich erwärmen an den grossen und bahnbrechenden Arbeiten der Vorfahren und der Zeitgenossen; man kann sie dem lebenden Geschlechte und namentlich der nachwachsenden Jugend als eine Leuchte des Strebens vorhalten» ${ }^{23}$.

Rudolf Virchow hatte mit seinem «Prinzip Zelle» allerdings nur scheinbar ein allseitig umfassendes Konzept vorgelegt. Dementsprechend hatte der Fortschritt auch seine Schattenseite. Es fällt nämlich auf, dass sich Virchow im Zusammenhang mit der Verwendung des Begriffes «Organismus» über den menschlichen Körper als Einheit nur vage äussert. Vielmehr zeigt sich die Tendenz, unverzüglich über die Eigenschaften und Funktionen der Organe und Körperteile zu berichten. Der «Organismus» hat scheinbar keine Eigenschaften, die ihm als Einheit direkt zugesprochen werden können. Offensichtlich lässt er sich nur im Rahmen seiner Bestandteile beschreiben und einordnen, «...denn erst in der Zelle finden die Molecüle ihre Zusammenfassung zu einer eigentlich lebendigen Einheit und aus Zellen setzt sich zuletzt jedes organische Wesen zusammen» $(1856)^{24}$. Entsprechend ist bei Virchow die Terminologie: «Zellen-Organismus», «zusammengesetzter Organismus», «Zusammenfügung sich gegenseitig beeinflussender Zellen zu einem Sammelorganismus» (1856) ${ }^{25}$. Virchow befindet sich damit zwangsläufig in Auseinandersetzung mit denen, «..., welchen die Zelle als einheitliche Grundlage des Lebens nicht genügt und welche daher irgend eine grössere, höhere Einheit suchen» ${ }^{26}$.

Diese Ansichten behält Virchow bei. Er äussert 1880: «Was den Arzt interessiert, das ist niemals eine Zelle, sondern immer eine Summe von Zellen, und die Krankheit im Sinne des practischen Arztes, im Sinne der speciellen

22 Ders., Krankheitswesen und Krankheitsursachen, S. 14/15; ders., Hundert Jahre Allgemeiner Pathologie, bes. S. 9/10 u. 17.

23 Ders., Herr Wunderlich, die Graeculi und die physiologische Heilkunde. Persönliches und Allgemeines, in: Archiv für pathologische Anatomie und Physiologie und für klinische Medicin 15 /N.F. 5 (1858) 393-400, hier S. 394.

24 Ders., Alter und neuer Vitalismus, S. 34 (n. 11). Siehe auch Virchows Ausführungen über die Zelle in: ders., Ernährungseinheiten und Krankheitsheerde, S. 375-399.

25 Ders., Alter und neuer Vitalismus, S. 50, 55 (n. 11).

26 Ebda., S. 34. 
Pathologie wird immer in dieser Weise verstanden werden müssen ${ }^{27}$. In diesem Zusammenhang klingt es eher beschwörend, wenn Virchow im Vorwort zu seinen «Gesammelten Abhandlungen zur wissenschaftlichen Medicin» beteuert, dass er sich niemals in der Lage befinden werde, «den Satz von der Einheit des menschlichen Wesens und seine Consequenzen zu verleugnen». In den folgenden Ausführungen zeigt sich vornehmlich der naturwissenschaftlich-organisch ausgerichtete Mediziner. Das Kapitel «Der Mensch» wendet sich gegen die «Transcendenz», in die das Menschengeschlecht durch «das unvollkommene, unwissenschaftliche Bewusstsein» getrieben wurde. Dem «Glauben» wird noch ein gewisses Eigenrecht zugestanden, der «Anthropomorphismus» wird abgelehnt: Die Möglichkeiten menschlichen Denkens, Fühlens und Wollens werden von Virchow im Hinblick auf das organische Korrelat beurteilt, die Möglichkeiten seines «sich Äusserns in der Welt» aus naturgesetzlicher Regelhaftigkeit abgeleitet. Das das wissenschaftliche Bedürfnis zur Annahme einer individuellen Seele führe, wird in Abrede gestellt ${ }^{28}$. In diesem Zusammenhang fällt ebenfalls auf, dass Virchows Definition von «Leben» sich vornehmlich auf die lebenden Einzelteile des Körpers stützt. Dabei wird das nicht wissenschaftlich Fassbare bewusst ausgeklammert: «Der Sitz des Lebens ist nicht identisch mit dem Sitz der Seele. Denn das Leben ist eine Eigenschaft aller Theile eines lebenden Organismus ... Für die heutige Betrachtung genügt es zu sagen, dass die Vorstellung von der Einheit des Lebens höherer Organismen unhaltbar geworden ist $»^{29}$. Letztlich muss konstatiert werden, dass die Darstellung des Individuums im Rahmen seines Krankheitsbegriffes seltsam farblos und blass bleibt. Ob es sich also bei Virchows Prinzip um einen fortschrittlichen Entwurf handelt, ist eine Frage des jeweiligen Standpunktes. Die geschilderten Defizite gaben schliesslich Anlass für eine neue Konzeption in der Pathologie.

27 Ders., Krankheitswesen und Krankheitsursachen, S. 8 (n. 13). Siehe auch Ders., Über die heutige Stellung der Pathologie, S. 90/91 (n. 12):«Wir sind in der Kenntnis der Zellelemente endlich an den Punkt gelangt, wo wir die wirklich tätigen Bestandteile des menschlichen Leibes vor uns haben, nicht mehr die geistigen, sondern die sichtbaren Elemente, die elementaren, die schaffenden, die tätigen Organismen».

28 Ders., Gesammelte Abhandlungen zur Wissenschaftlichen Medicin, $<$ Kapitel: Der Mensch, Das Leben, Die Medicin, Die Krankheit, Die Seuche>, S. 1-56, Frankfurt/M. 1856; Zitate: Vorwort/S. 5. Der Nachweis Davids, Virchows Vorstellungen zum Menschen sei von der Einheit des Organismus geprägt gewesen, stützt sich fast ausschliesslich auf diese Quelle, was wie gezeigt unzureichend ist. Die anderen von David angeführten Zitate bezeugen letztlich Virchows Anschauung des Organismus als Produkt seiner Teile. Vgl. David, Rudolf Virchow und die Medizin des 20. Jahrhunderts, S. 218-220. Zu politisch-biologischen Analogien bei Virchow und in diesem Zusammenhang zum Organismus-Begriff vgl. Renato G. Mazzolini, Politisch-Biologische Analogien im Frühwerk Rudolf Virchows, Marburg 1988, S. 23-32.

29 Rudolf Virchow, Die Continuität des Lebens als Grundlage der modernen biologischen Anschauung, in: Archiv für pathologische Anatomie und Physiologie und für klinische Medizin 150 (1897), 14. Folge Bd. 10, H. 1, S. 4-15, hier S. 6/7. 


\section{Die Konstitutionspathologie}

Seit dem letzten Drittel des 19. Jahrhunderts meldete sich aus unterschiedlichen Gründen verstärkt Kritik an der Zellularpathologie zu Wort. Ein massgeblicher Grund ist sicherlich im Aufschwung der Bakteriologie zu sehen, da mit der Entdeckung von Mikroben eine Diskussion um die Krankheitsursachen einsetzte - ein Thema, das in der Zellularpathologie eher eine Randstellung einnahm. Die nach wie vor nicht eindeutig zu beantwortende Frage nach der Kausalerklärung menschlicher Krankheiten, die Frage nach internen oder externen Ursachen, führte letztlich zur Entwicklung «konditionalistischer» Theorien, die ein komplexes Bedingungsgefüge für die Krankheitsgenese verantwortlich machten (Verworn, v. Hansemann) ${ }^{30}$. Von hier aus war es nur ein kleiner Schritt zum Entwurf einer «Konstitutionspathologie», deren Ursprünge sich bis in das 19. Jahrhundert verfolgen lassen. Ihren Höhepunkt erlebte diese Richtung, die im Rahmen der allgemeinen «Konstitutionslehre» in gegenseitiger Beeinflussung thematisch sehr eng mit zeitgenössischen Strömungen der klinischen Medizin verbunden war, vor allem in den 20er und 30er Jahren unseres Jahrhunderts ${ }^{31}$. Dabei entwickelte die Konstitutionslehre und damit auch die Konstitutionspathologie in Deutschland ihr eigenes Profil, in den angelsächsischen Ländern verschaffte sie sich schwerer und später Gehör ${ }^{32}$.

Die Konstitutionspathologie legte das Schwergewicht ihrer Forschung auf die Eigenheiten des Gesamtorganismus und tat dies in bewusster Anschauung der Lücke, die die Zellularpathologie hinterlassen hatte. Letztere bildete den Hintergrund, um - mit den Worten des Pathologen Felix Marchand (1846-1928) - «an die Stelle der Zellularpathologie eine Konstitutionspathologie zu setzen, die auf dem Ineinandergreifen, den Korrelationen, der ge-

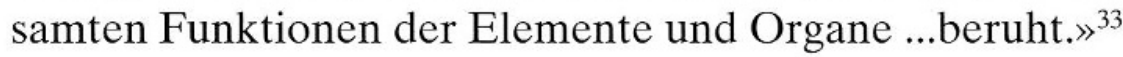

Wichtig wurden vor allen Dingen der Einbezug der Umwelt und der Einbezug genetischer Forschungen, d.h. die Erträge der Vererbungslehre.

30 Vgl. die ausführliche Darstellung der Diskussion zwischen «Kausalisten» und «Konditionalisten» in: Dietrich v. Engelhardt, Kausalität und Konditionalität in der modernen Medizin, in: Pathogenese. Grundzüge und Perspektiven einer Theoretischen Pathologie, hrsg.v. Heinrich Schipperges, Berlin/Heidelberg/New York/Tokyo 1985, S. 32-58.

31 Erste Umrisse einer Geschichte der Konstitutionslehre und auch -pathologie bei Johannes Probst,Zur Entwicklung der Konstitutionslehre zwischen 1911 und 1980, Diss.Med.Fak. Freiburg 1982. Im Zusammenhang mit der Konstitutionspathologie siehe vor allem die Seiten 1 bis 44 .

32 Ebda., S. 1. Siehe auch Manfred Koch, Elfriede Höhn, Die Entwicklung der Konstitutionsforschung in den angloamerikanischen Ländern, in: Fortschritte der Neurologie, Psychiatrie und ihrer Grenzgebiete 21 (1953), H. 8, S. 355-391, hier bes. S. 355.

33 Felix Marchand, Zum hundertsten Geburtstag von Rudolf Virchow, in: Münchener Medizinische Wochenschrift 68 (1921), Nr. 40, S. 1271-1274, hier S. 1273. 
Robert Rössle (1876-1956), ein Nachfolger Virchows auf dem Lehrstuhl für Pathologie an der Berliner Charité, fasste 1921 die Beziehungen der Zellularpathologie zur Konstitutionspathologie noch einmal zusammen, indem er auf die durch Virchow gelegten Grundlagen auch in Hinsicht auf konstitutionelle Einflüsse auf die Genese von Erkrankungen des Menschen hinwies und die «Organismusidee» und die «Ätiologie» als Schwerpunkte der neuen Richtung umriss ${ }^{34}$. Dabei wies er auf das Fehlen ausreichender Kenntnisse in bezug auf die Vererbungslehre hin. Vor allem stecke die Konstitutionspathologie noch in den Kinderschuhen, da es ihr bis heute nicht gelänge, umweltbedingte Störungen von genetisch verursachten zu trennen. Aus dieser Erkenntnis heraus entwickelt Rössle die Forderung nach der genealogischen Untersuchung ganzer Familien, wobei über vergleichende Organuntersuchungen hinaus die Ursache für kombinierte Störungen in einem oder in mehreren Organen gefunden werden sollen: «Und wenn wir damit selbstverständlich über den Rahmen der Organpathologie hinauskommen, so bleiben wir doch und müssen bleiben in demjenigen der Zellularpathologie Virchows ${ }^{35}$. Dennoch betrachtete Rössle die Konstitutionspathologie durchaus als progressive Richtung seines Faches: «In diesem Sinn sehen wir Virchows Lehre lebenskräftig und weitblickend genug, um auch noch denjenigen Fortschritten der heutigen Medizin sich anzupassen, denen gegenüber der reine Lokalisationsgedanke als solcher versagt, das sind 2 Gebiete: erstens die Lehre von den Korrelationen und zweitens die Lehre von der Vererbung ${ }^{36}$.

Trotz des Versuches, Lücken in der Virchowschen Pathologie zu füllen, stand allerdings nach wie vor das morphologische Substrat im Vordergrund der Betrachtung von Krankheit. Die Konstitutionspathologie blieb daher einem gewissen Lokalismus verpflichtet, wiewohl die Auswirkungen einer lokalen Schädigung auf den Körper als Ganzheit mitberücksichtigt wurden: «Krankheit ist ein Vorgang, der sich in einzelnen Teilen des Körpers, in Organen, Geweben und Zellen abspielen kann, aber den Gesamtorganismus bei der weitgehenden wechselseitigen Abhängigkeit in hohem Masse mittrifft ${ }^{37}$. Diese Beurteilung betraf sowohl die inneren als auch die umweltbedingten Ursachen von Leiden. Fortschritte der Pathologie - im Sinne der Konstitutionspathologie die Erweiterung von Virchows Prinzip in Richtung einer ganzheitlichen, d.h. hier gesamtorganismischen Sehweise - stiessen an dem geschilderten Punkt an ihre Grenzen.

34 Robert Rössle, Rudolf Virchow und die Konstitutionspathologie, in: Münchener Medizinische Wochenschrift 68 (1921), Nr. 40, S. 1274-1277, hier vor allem S. 1274/1275.

35 Ebda., S. 1276/1277, Zitat S. 1277.

36 Ebda., S. 1275.

37 Albert Dietrich, Grundriss der Allgemeinen Pathologie, Leipzig 1927, S. 4. 


\section{Molekularpathologie und Theoretische Pathologie}

In den 50er Jahren unseres Jahrhunderts erschlossen sich vor allem durch die Entwicklung des Elektronenmikroskops, das den Blick auf die subzelluläre Ebene lenkte und damit noch mehr «verengte», für den Pathologen neue Forschungsmöglichkeiten. Ganzheitliche Betrachtungsweisen des Menschen traten hierdurch tendenziell wieder in den Hintergrund. Es war nunmehr möglich, die Ultrastruktur der Zelle, wie beispielsweise die Zellorganellen, zu untersuchen. Dabei konnten nicht nur die einzelnen Zellbestandteile weiter differenziert werden, sondern auf der Grundlage derartiger Ausdifferenzierungen die Ursachen einzelner Erkrankungen in bestimmten Bausteinen der Zelle ermittelt werden. Mit der Entwicklung des Konzeptes der «Organellopathien» ging die immer stärkere Inanspruchnahme biochemischer Verfahren zu Forschung und Diagnostik einher. Dadurch war es möglich, neue Erkenntnisse über die Verbindungen von Funktion und Struktur in der Zelle zu gewinnen - die Bedeutung der pathologischen Physiologie hatte Virchow immer wieder gefordert. Diese Forderung - so scheint es - konnte erst verspätet erfüllt werden ${ }^{38}$. Ihre Einlösung bedeutete gleichfalls eine Rückkehr zum strengen Lokalismus Virchows, wobei erneut die Kenntnis des Details grossgeschrieben wurde. Dies wurde wiederum auch als Fortschritt betrachtet. Martin Nordmann (1895-1980), Prosektor am Pathologischen und Bakteriologischen Institut Hannover, äusserte in seiner Eigenschaft als Vorsitzender der Deutschen Gesellschaft für Pathologie auf der 40. Tagung in Düsseldorf 1956: «Aber allein der Einbau der ultramikrooptischen Befunde in die pathologische Histologie wird eine Generation von Forschern beschäftigen. Ich bin dabei glücklich wie Moses beim Blick in das gelobte Land ${ }^{39}$.

Die Pathologie sieht sich heute zwar nach wie vor dem Erbe Virchows verpflichtet, und die Zelle als Grundbaustein des Lebens steht weiterhin im Mittelpunkt pathologischer Forschung ${ }^{40}$. Neben den morphologischen ist aller-

38 Zur heutigen Pathologie vgl. David, Rudolf Virchow und die Medizin des 20. Jahrhunderts, vor allem S. 47-58; Franz Büchner, Entwicklungen und Grenzen der Cellularpathologie, in: Klinische Wochenschrift 33 (1955), H. 13/14, S. 289-294; Volker Becker, 10 Jahre «Der Pathologe», in: Der Pathologe 10 (1989) 1/2; Robert Fischer, Die Wissenschaftlichkeit in der Pathologie - Die deutschsprachige Pathologie im Spannungsfeld ihrer Gesellschaften und Tagungen, in: Verhandlungen der Deutschen Gesellschaft für Pathologie, 76. Tagung 1992 in Graz, Stuttgart 1992, S. XXXVIII-XLIV (1992).

39 Vgl. Martin Nordmann, Eröffnungsrede des Vorsitzenden, in: Verhandlungen der Deutschen Gesellschaft für Pathologie, 40. Tagung 1956 in Düsseldorf, Stuttgart 1956, S. 5-7, Zitat auf S. 7. Siehe auch Volker Becker, Wilhelm Doerr, Heinrich Schipperges, Krankheitsbegriff und Krankheitsforschung im Lichte der Präsidialansprachen der Deutschen Gesellschaft für Pathologie (1897-1992), Stuttgart/Jena 1993, S. 95/96.

40 Vgl. dazu Joachim Kracht, Differenzierung der Pathologie. Eröffnungsrede des Vorsitzenden, in: Verhandlungen der Deutschen Gesellschaft für Pathologie, 72. Tagung in Hannover, Stuttgart/Jena 1988, S. XXXIII-XLIII, hier S. XXXIII+XLII. 
dings mit Macht der «biochemische Gedanke» getreten ${ }^{41}$. Obwohl durch den Aufschwung der Biochemie im Fach die Möglichkeit gegeben ist, dass der morphologische Gedanke immer mehr in den Hintergrund tritt ${ }^{42}$, so ist in unserem Zusammenhang wichtig, dass beide Bereiche dem Lokalismus verhaftet sind und eine übergreifende Gesamtschau nicht eben fördern ${ }^{43}$.

Als Reaktion auf den beschriebenen Prozess der erneuten, verstärkten lokalistischen Betrachtung des kranken Individuums im Rahmen von Diagnostik und Therapie fanden seit den 60er Jahren in Heidelberg Bemühungen um die Errichtung einer «Theoretischen Pathologie» statt, die, ebenfalls mit der Hoffnung auf Fortschritte ${ }^{44}$, in die Gründung einer eigenen Forschungsstelle einmündeten. Ziel war es, den Begriff der Krankheit wieder stärker auf die Gesamtpersönlichkeit des Menschen anzuwenden, indem man sie aus der Sicht der Pathologie als die «Gesamtheit der aus der Variationsbreite gestaltlicher und funktioneller Lebensäusserungen herausfallenden Erscheinungen $»^{45}$ begriff. Erkenntnistheoretische, aber auch anthropologische Momente - d.h. beispielsweise die Erfahrung von Krankheit, Schmerz, Tod - sollten wieder ein stärkeres Gewicht nicht nur in der Pathologie, sondern von ihr ausgehend in der gesamten Medizin erhalten. Nicht zuletzt durch die Einbeziehung des Intrapsychischen und des Leib-SeeleProblems griff die «Theoretische Pathologie» weit über die streng morphologisch ausgerichtete Ganzheitsmedizin der «Konstitutionspathologie» hinaus.

Gerade im Hinblick auf die Problematik der heutigen Medizin war auch das Eintreten für eine stärkere Akzeptanz von Krankheit vor allem im Falle ihres chronischen Charakters und die Forderung an den Arzt, nicht nur Therapeut, sondern auch kontinuierlicher Berater und Krankheitsbegleiter zu

41 David, Rudolf Virchow und die Medizin des 20. Jahrhunderts, S. 47-58 (n. 38).

42 Die anatomische Grundlage seiner Arbeit kann der Pathologe durch die Beschränkung seiner Aufmerksamkeit auf isolierte Stoffwechselprozesse aus dem Auge verlieren. Pathologische Veränderungen werden aber nach wie vor auch an der veränderten Morphologie abgelesen: Sektionen sind nach wie vor ein unverzichtbarer Bestandteil der wissenschaftlichen Medizin. Vgl. dazu Hans-Werner Altmann, Die Pathologie an der Schwelle des neuen Jahrhunderts, in: Würzburger medizinhistorische Mitteilungen 8 (1990) 351-368; Dieter Modelmog, Roland Goertchen, Der Stellenwert von Obduktionsergebnissen in Beziehung zu Sektionsfrequenz und amtlicher Todesursachenstatistik, in: Deutsches Ärzteblatt 89 (1992), H. 42, S. 2174-2177.

43 Zur heutigen Pathologie siehe auch Harold E. Bowman, Marjorie J. Williams, The Practice of Pathology into the 21st Century: Introduction, in: Archives of Pathology and Laboratory Medicine 110 (April 1986) 261-263. Zur historischen Einordnung des Faches durch die Pathologie, hier bezogen auf die Verhältnisse in den USA, vgl. William W. McLendon, A Historical Perspective as a Compass for the Future of Pathology, in: ebda., S. 284-288.

44 Wilhelm Doerr, Vorbemerkung: Entwicklungsgeschichte des Vorhabens, in: ders., Heinrich Schipperges (Hrsg.), Was ist theoretische Pathologie?, Berlin/Heidelberg/New York 1979, S. 1-3, hier S.3.

45 Ders., Aus der Sicht der konventionellen Pathologie, in: ebda., S. 4-20, hier S. 11. 
sein, entscheidend. Dies bedeutete gleichzeitig eine grundlegende Einschränkung des auf die Heilung reduzierten Fortschrittsbegriffes der Schulmedizin. Die Akzeptanz von Krankheit als einem Grundphänomen des menschlichen Lebens und das Gespräch mit dem Kranken stehen dem fortschrittsorientierten Fahnden nach neuen wirksamen Therapieformen in diesem Konzept durchaus gleichberechtigt zur Seite ${ }^{46}$.

\section{Fortschritte in der Pathologie? - Bemerkungen zu einem Problem}

Die moderne Pathologie steht weiterhin eindeutig zu einer fortschrittsorientierten Sehweise. Ähnlich wie bereits Virchow wird der heutige Standort in historischem Licht betrachtet und die Ergebnisse des Faches unter Aufzeichnung einer linearen Entwicklung als quasi notwendig legitimiert. Dies lässt sich logisch begründen, denn die Medizin hat nicht zuletzt infolge der Konzepte der Pathologie und ihrer Entwicklung seit der Mitte des letzten Jahrhunderts einen entscheidenden Durchbruch erzielt: Die therapeutische Effektivität hat in vielen Bereichen tatsächlich zugenommen. Wenn durch die Aufdeckung molekularer Zellfunktionen in letzter Konsequenz ein Beitrag zur Krankheitsbekämpfung geleistet werden kann, handelt es sich aus ärztlich-medizinischer Sicht tatsächlich um eine Effektivitätssteigerung. Dementsprechend kann man also von Fortschritt im Sinne der «Verbesserung» der medizinischen Leistung sprechen ${ }^{47}$. Kann man aber deshalb die generelle Entwicklung der Pathologie und in ihrem Gefolge der naturwissenschaftlichen Medizin als «fortschrittlich» bezeichnen? Damit ist das Problem der Bemessungsgrundlage von «Fortschritt» überhaupt angesprochen. Hier ergeben sich deutliche Schwierigkeiten. Von der Bevölkerung wurden die praktischen Konsequenzen des lokalistischen Prinzips - man denke in diesem Zusammenhang an den Aufstieg der Naturheilkunde seit den letzten Dekaden des 19. Jahrhunderts - anscheinend nicht ohne weiteres nur als po-

46 Zur «Theoretischen Pathologie» siehe auch: Wilhelm Doerr, Ars longa - vita brevis. Problemgeschichte kritischer Fragen II, Berlin u.a. 1991; Wilhelm Doerr, Heinrich Schipperges (Hrsg.), Modelle der Pathologischen Physiologie, Berlin u.a. 1988; Wilhelm Doerr, Georg Benno Gruber, Problemgeschichte kritischer Fragen. Angeborene Herzfehler. Schlagaderdifformitäten-Krankheitsbegriff-Homologieprinzip-Ethik, Berlin u.a. 1988; Wilhelm Doerr, Walter Hofmann, A.J. Linzbach, K.Rother, F. Seitelberger, Neue Beiträge zur Theoretischen Pathologie, hrsg.v. Heinrich Schipperges, Berlin u.a. 1981; V. Becker, K. Goerttler, H.H. Jansen (Hrsg.), Konzepte der Theoretischen Pathologie, Berlin u.a. 1980.

47 Siehe hierzu auch H.-J. Staudinger, Naturwissenschaft, in: Seidler (Hg.), Wörterbuch medizinischer Grundbegriffe, S. 222-226, hier vor allem S. 225. 
sitiv empfunden ${ }^{48}$. «Fortschritt» lässt sich dementsprechend in einer Beschränkung auf medizinisch-naturwissenschaftliche Prämissen nicht ohne Einschränkungen postulieren, auch wenn bei aller Diskussion um die Ganzheitsmedizin theoretische Postulate eines anthropologisch-philosophischen Menschenbildes immer noch der praktischen Umsetzung harren.

Dies führt unweigerlich zur Betrachtung der «Fortschrittsfrage» als «Sinnfrage»: Wozu Fortschritt in der Medizin und wem soll er dienen? Die naheliegende Antwort, er solle das Leiden der Menschen lindern und er sei im Falle der Pathologie und damit der Medizin notwendig zum Wohl der Patienten, führt wiederum zum Rückgriff auf die Arzt-Patient-Beziehung, auf die von Eduard Seidler betonte «Primärerfahrung von Not und Hilfe» als einer Grundkonstante der Medizin und damit auch der Medizingeschichte. Die «Primärerfahrungen des Missbefindens» und die daraus abgeleiteten «Grundmodelle der Bewältigung» sind konstante Erlebens- und Befindensweisen vor jeder naturwissenschaftlich-exakten Durchdringung ${ }^{49}$. Dieser Zustand ist weiterhin dadurch charakterisiert, dass das Erleben des Patienten durch seine Umwelt und die vielfältigen Bezüge zu dieser geprägt wird und er dies auch in das Zwiegespräch mit dem Arzt einbringt. Die Sensibilität für die grundsätzlichen Phänomene des Krankwerdens in enger Beziehung zur sozialen Umwelt des Patienten erfordern grundsätzlich eine auch geisteswissenschaftliche Ausrichtung des Arztes. Bedenkt man ferner, dass auch der Arzt ein Mitglied der menschlichen Gemeinschaft ${ }^{50}$ ist und - dies wird seit etwa 90 Jahren wissenschaftstheoretisch untersucht - die Sozialbezüge des Forschers auch im Institut und im Labor ihre Wirksamkeit entfalten ${ }^{51}$, ferner auch rein erkenntnistheoretisch nur sehr begrenzt Wissen von dem, was wir als Realität wahrnehmen, durch den forschenden Mediziner erlangt werden

48 Beispielhaft für die Reaktion der Ärzteschaft auf die aufstrebende Naturheilkunde: Georg Sticker, Über Naturheilkunst. Vier Reden, Giessen 1909.

$49 \mathrm{Vgl}$. Eduard Seidler, Primärerfahrung von Not und Hilfe, in: Krankheit. Heilkunst. Heilung, hrsg.v. Heinrich Schipperges, Eduard Seidler und Paul U. Unschuld (Veröff. d. «Inst. f. Hist. Anthrop. E.V.», Bd.1), S. 399-418; ders., Modelle der Arzt-Patient-Beziehung, in: Der Arzt als Begleiter (Herrenalber Texte 25), Karlsruhe 1980, S. 11-26, hier S. 11-17; ders., Krankheit und Gesundheit, in: ders. (Hg.), Wörterbuch medizinischer Grundbegriffe, S. 172-182.

50 Karl Eduard Rothschuh spricht in diesem Zusammenhang von bereichsfremden (religiöse, philosophische, politische etc.) und bereichseigenen (Theorie und Praxis der Medizin) Vorstellungen eines Zeitalters, die den Arzt prägen; vgl. ders., 1. Einleitung: Konzepte der Medizin. Bedürfnis, Begriff, Formen, in: ders., Konzepte der Medizin in Vergangenheit und Gegenwart, Stuttgart 1978, S. 1-20, hier S. 10.

51 Vgl. hierzu Peter Moraw, Vom Lebensweg des deutschen Professors, in: Forschung. Mitteilungen der DFG 1988, H. 4. 
$k^{2} n^{52}$, so zeigt sich klar die Relativität eines rein naturwissenschaftlichen Heilungsansatzes, der sich auf das morphologische Substrat beschränkt.

Im Folgenden soll die These aufgestellt werden, dass die geschilderte Problematik in der zweiten Hälfte des 19. Jahrhunderts verschärft auftrat und unter anderem durch eine unterschiedlichem Denken entspringenden Kommunikationsstörung zwischen Arzt und Patient verursacht wurde. In Anlehnung an die von Josef N. Neumann geäusserten Gedanken zur Handlungslegitimation ärztlicher Praxis ${ }^{53}$ kann m. E. die These aufgestellt werden, dass die direkte Übernahme logischer Beweisketten aus den Labors der theoretischen und klinischen Institute unter dem Primat des «Machbarkeitskriteriums» in die ärztliche Behandlung erfolgte. Dies geschah infolge der Durchsetzung des lokalistischen Zellprinzips in Kombination mit der naturwissenschaftlich-kausalanalytischen Methodik. Dabei wurde es versäumt, dieses Konstrukt der Forschung nochmals der Realität an der medizinischen Basis der Krankenversorgung anzupassen - eine Notwendigkeit, da der Forscher zwar die Konstruktion des Wissens und deren Umfeld, nicht aber Umsetzung in der Praxis überblicken $\mathrm{kann}^{54}$.

Der Anspruch, eine wissenschaftliche Hypothese formal-logisch auf deren «Richtigkeit» und damit deren Anwendbarkeit zu überprüfen, um sie anschliessend auch umzusetzen, entstand mit den exakten Wissenschaften im 19. Jahrhundert. Entscheidend dabei ist, dass die einwandfreie Logik in der Beweisführung für die praktische Umsetzung ausreichte, dass dem wissenschaftlichen Experiment unzugängliche Faktoren nicht mehr berücksichtigt zu werden brauchten. Das nach 1850 gestiegene soziale Ansehen der Medizin und ihrer Vertreter erleicherte dabei die geradlinige Handhabung von experimenteller Forschung zu therapeutischen Zwecken, der Einführung der in Labor und Sektionssaal gewonnenen logischen Erkenntniszusammenhänge in die Praxis. Der durch die pathologische Anatomie und ihre Vordenker inaugurierte Lokalismus bot die Grundlage, um den Denkhorizont des Arztes auf Zellverbände und Organe zu beschränken ${ }^{55}$.

$52 \mathrm{Zu}$ den Sozialbezügen des Forschenden: a) für den mikrosoziologischen Bereich: Karin Knorr-Cetina, Die Fabrikation von Erkenntnis. Zur Anthropologie der Naturwissenschaft, $<$ Oxford 1981> Frankfurt/M. 1991. Siehe hier vor allem das Vorwort von Rom Harré, S. 11-14; b) für den makrosoziologischen Bereich: Thomas S. Kuhn, Die Struktur wissenschaftlicher Revolutionen (1962), Frankfurt/M. ${ }^{10} 1989$. Zur Erkenntnistheorie: Karl R. Popper, Logik der Forschung, Tübingen 1966.

53 Josef N. Neumann, Die Ganzheit des Menschen als Herausforderung an Spezialisierung und Handlungslegitimation medizinischer Praxis, in: Freiburger Universitätsblätter 31 (1992), H. 118, S. 41-58.

54 Siehe hierzu die Ausführungen Eduard Seidlers zum «Problem des Machbaren», in: ders., Modelle der Arzt-Patient-Beziehung, S. 23-26. 


\section{Schluss}

Handelt es sich um einen fortschrittlichen Prozess, den die Pathologie seit 1858 durchgemacht hat? Mit der Bewertung, es handele sich um Fortschritt, aber wie jeder Fortschritt, sei er eben auch ambivalent und habe seine Kehrseiten, kann man sich nicht zufriedengeben ${ }^{56}$. Ebensowenig kann man für die Medizin eine Fortschrittsentwicklung postulieren mit dem Hinweis, bestimmte geisteswissenschaftliche Bereiche in der Heilkunde entzögen sich einer solchen Beurteilung ${ }^{57}$.

Da sich die gestellte Frage für die Pathologie nicht ohne weiteres bejahen lässt, sollte man angesichts des dargestellten Verlaufes der Fachgeschichte nicht von einem fortschrittlichen Wandel in Richtung auf einen vermeintlich optimalen Endzustand sprechen, sondern vielmehr von einem «fortschreitenden Prozess» im nichtwertenden Sinne, in dessen Rahmen auf bestehende Probleme und Herausforderungen reagiert wurde und wo man gleichzeitig versuchte, sie in dem jeweiligen historischen Zeitabschnitt zu lösen. In diesem Zusammenhang plädiert der vorliegende Text zudem für eine Zerlegung der normativen Prädikatszuweisung «Fortschritt» in die Einzelbereiche, in denen ein Konsens über das Vorhandensein eines solchen erzielt werden kann. Im Falle der Pathologie und ihrer Gründerfunktion für die naturwissenschaftliche Heilkunde sind die Forschung in der Pathologie und ihre theoretischen Konzepte von deren praktischer Umsetzung und der Auffassung der daran beteiligten Menschen zu trennen - auch wenn sich die Personen nicht immer ohne weiteres einem Bereich zuordnen lassen. Was für den $\mathrm{Pa}-$ thologen auf der Grundlage streng lokalistischer Anschauung Fortschritt bedeutet, muss noch lange nicht für den behandelnden Arzt oder Patienten gelten, die beide meist nicht in diesem Ausmass über den Krankheitsbegriff und das Krankheitswesen reflektieren, sondern mit dessen Umsetzung befasst sind.

55 Es sei darauf hingewiesen, dass nicht nur in der Medizin, sondern auch in den Rechtswissenschaften im Deutschland des 19. Jahrhundert die reibungslose Umsetzung theoretisch-logischer Sachverhalte in die Praxis als möglich angesehen wurde. Siehe hierzu: Gerhard Dilcher, Das Gesellschaftsbild der Rechtswissenschaft und die soziale Frage, in: Klaus Vondung (Hg.), Das wilhelminische Bildungsbürgertum. Zur Sozialgeschichte seiner Ideen, Göttingen 1976, S. 53-66, bes. S. 55.

56 Dies ist beispielsweise der Tenor in: Iago Galdston, The pathogenicity of progress. An essay on medical historiography, in: Medical History 9 (1965) 127-132.

57 Eine solche naturwissenschaftlich geprägte Argumentation findet sich in dem jüngst erschienenen Aufsatz zur Fortschrittsproblematik von Wolfgang Gerok, Probleme des Fortschritts aus der Sicht des Arztes und Forschers, in: Freiburger Universitätsblätter 31 (1992), H. 115, S. 129-139, hier S. 130. 
Auf der Suche nach den Bedingungen, welche die Bewertung einer Innovation oder eines Zustandes als Fortschritt vornehmen, stösst man auf drei Faktoren, nämlich

1. das Subjekt und seine Einstellung zum betreffenden Gegenstand und zu seinem Umfeld,

2. den Gegenstand oder Sachverhalt selbst,

3. die historische Situation, in der die Entscheidung - Fortschritt:ja oder nein - gefällt wird.

Von diesen drei Faktoren ist die Entscheidung über etwaige Fortschrittlichkeit abhängig. Es ist demnach keine objektive Entscheidung: Die Menschen, die über Progressivität urteilen, tun dies auf der Basis ihrer jeweiligen Mentalität. Ferner sind sie in ihren Zeitkontext eingebunden, der in der Regel nur bestimmte Variationen des Blickwinkels zulässt. Die Qualität des Gegenstandes - bieten sich durch ihn neue ausbaufähige Möglichkeiten? - entscheidet schliesslich über die Beantwortung der Frage mit.

Aus diesen Ausführungen wird zudem deutlich, dass Fortschritt zur Konsensfrage unter den Menschen wird, wenn eine allgemeine Entscheidung über die Progressivität eines Sachverhaltes oder Gegenstandes gefällt werden soll. Wichtig wird nicht nur der Konsens zwischen Pathologe und Praktiker, sondern auch zwischen Praktiker und Patient, dessen Unbehagen gegenüber der Entwicklung der Medizin zum Teil die theoretischen Diskussionen seit der zweiten Hälfte des 19. Jahrhunderts ausgelöst hat, wobei zwischen den Ebenen immer wieder die «Sinnfrage» gestellt werden muss. So kommt man zurück auf die Grundkonstante von «Not und Hilfe», zum eigentlichen «Sinn» der Denkentwürfe über das Wesen von Krankheit. An dieser Stelle liegt die Verbindung von Theorie und Praxis, die Umsetzung der Theorien über Krankheit und die Art und Weise der Bekämpfung von Leiden. Allgemein als solcher akzeptierter Fortschritt würde durch einen breiten Konsens entstehen. Dieser kommt nur zustande auf der Grundlage der gegenseitigen Anerkennung der Lebenswirklichkeiten, in denen sich die handelnden Patienten, Ärzte und Forscher befinden ${ }^{58}$. In diesem Zusammenhang könnte die Vermittlung der theoretischen Entwürfe einer Pathologie, die sich mit dem von ihr entwickelten Lokalismus auseinandersetzt und unter Berücksichtigung von sozialer und Umweltsituation auf den Einzelfall angewandt wird, eine nicht unbedeutende Rolle spielen.

58 Ansätze hierzu: ebda., S. 132-139. 